\title{
RELACIÓN ENTRE CALIDAD DE VIDA EN EL TRABAJO Y ROLES DE GÉNERO EN DOCENTES UNIVERSITARIOS
}

\section{RELATIONSHIP BETWEEN QUALITY OF WORK LIFE AND GENDER ROLES IN UNIVERSITY TEACHERS}

\author{
Christian Zambrano ${ }^{1}$ \\ Sonia Maritza Matabanchoy ${ }^{2}$ \\ Universidad de Nariño, Pasto, Colombia.
}

\section{RESUMEN}

La Calidad de vida en el trabajo (CVL) se entiende como un proceso dinámico y continuo que permite el desarrollo integral del colaborador, en el que se articulan aspectos objetivos y subjetivos e intervienen aspectos ambientales, culturales, educativos y sociales. En este sentido, variables como los Roles de género designan funciones, expectativas y normas que se esperan que mujeres y hombres cumplan en los diversos contextos de interacción, como el laboral, por lo que determinados Roles de género pueden influir en el trabajador, en las 1 cazambranopsi@gmail.com, Docente investigador Universidad de Nariño, PhD. en Ciencias de la Educación (c) Universidad de Nariño. Christian Zambrano: https://orcid. org/0000-0001-8482-0345

2 somapsicologa@yahoo.es, Docente investigadora Universidad de Nariño, PhD. En Psicología Universidad de Nariño. Sonia Matabanchoy: https://orcid.org/00000002-3681-8440 funciones que se desempeña y en el desarrollo de sus competencias.

Considerando que, en las universidades, los docentes desde su rol profesional y de género, se exponen a condiciones que pueden afectar la ejecución de su trabajo, salud y bienestar; la presente investigación, tuvo por objetivo analizar la relación entre Calidad de vida en el trabajo y Roles de género en docentes adscritos a una universidad pública de la ciudad de San Juan de Pasto (Colombia). Para este fin, se partió de un paradigma cuantitativo correlacional con un diseño transversal.

Los principales resultados indican en los docentes universitarios un nivel medio de CVL, una percepción considerablemente estereotipada en Roles de género, y una relación débil entre las dos variables objeto de estudio y sus dimensiones. 
PALABRAS CLAVE: Calidad de vida laboral, roles de género, docentes universitarios.

\section{ABSTRACT}

Quality of Work Life (QWL) is understood as a dynamic and continuous process that allows the integral development of the employee, in which objective and subjective aspects are articulated and environmental, cultural, educational and social aspects intervene. In this sense, variables such as Gender Roles designate functions, expectations and norms that women and men are expected to fulfill in the various contexts of interaction, such as work, so that certain gender roles can influence the worker, in the functions performed and in the development of their competencies.

Considering that in universities, teachers from their professional and gender roles are exposed to conditions that can affect the execution of their work, health and well-being; the objective of this research was to analyze the relationship between Quality of life at work and gender roles in teachers assigned to a public university in the city of San Juan de Pasto (Colombia). For this purpose, we started from a correlational quantitative paradigm with a cross-sectional design.

The main results indicate in university teachers a medium level of QWL, a considerably stereotyped perception in gender roles, and a weak relationship between the two variables under study and their dimensions.

KEYWORDS: Quality of Work Life, Gender Roles, university teachers.

\section{INTRODUCCIÓN}

La Declaración Mundial sobre la Educación Superior en el Siglo XXI permite visualizar los desafíos y dificultades a los que se enfrenta la educación, relacionados con temas de financiación, igualdad de condiciones de acceso, mejor capacitación del personal, calidad en la enseñanza, entre otras; que, a su vez, retan a educar, formar y forjar una nueva visión de educación superior (UNESCO, 1998).

En ese sentido, es preciso anotar que más allá de asumir el reto de la capacitación del personal docente como un desafío, es importante resaltar del importante rol que desempeña el docente desde su integralidad como ser humano en la labor, en el desempeño de sus funciones y en el impacto en la educación. Si bien los docentes universitarios del Estado en Colombia cuentan con un marco normativo claro que define aspectos relacionados con procesos de administración del talento humano como son la vinculación, incentivos y compensación, se hace necesario seguir trabajando con procesos de desarrollo del talento humano relacionados con salud y bienestar, que tienen implicaciones directas en la calidad de vida laboral o calidad de vida en el trabajo (CVL). Solo así, se aborda de manera integral el desarrollo del ejercicio docente, cumpliendo con las tres dimensiones que la conforman: disciplinar, pedagógica y humana (Clavijo y Balaguera, 2020; Salas, et. al, 2021).

Abordar al docente como trabajador implica comprender también los riesgos a los que se expone en ejecución de su trabajo. En ese sentido, la Primera Encuesta Nacional de Condiciones de Salud y Trabajo en el Sistema General de Riesgos profesionales en Colombia (Ministerio del Trabajo, 2007), hace evidente como los riesgos físicos (ruido, iluminación inadecuada, temperatura poco confortable), riesgos ergonómicos (movimientos repetitivos o posturas sostenidas) y riegos psicosociales (atención al público y aspectos relacionados con violencia laboral), tienen implicaciones directas en la salud física y mental del docente.

De este mismo modo, en la segunda en la Segunda Encuesta Nacional de Condiciones de Seguridad y Salud en el Trabajo en el Sistema 
General de Riesgos en Colombia (Ministerio del Trabajo, 2013), se reitera la necesidad de intervenir sobre los factores de riesgo antes mencionados y se adiciona la importancia de fortalecer los programas de seguridad y salud en el trabajo de manera completa e integral. Este tipo de evidencias dan cuenta de la necesidad de fortalecer e impulsar el desarrollo de programas de promoción y prevención que contribuyan al mejoramiento de aspectos objetivos relacionados con la CVL del docente.

Ahora bien, para centrarse en aspectos que convocan la mirada de la psicología de salud ocupacional en la labor del docente, se encuentra los factores de riesgo psicosocial, que de acuerdo Protocolo de intervención de factores psicosociales en el sector educativo del Ministerio del Trabajo (2019) en Colombia, se reportan las siguientes:

Demandas ambientales y de esfuerzo físico (jornadas extensas de trabajo en el que su mayor parte, el docente permanece de pie, de igual forma movimientos repetitivos de miembro superior) y demandas emocionales y de expresión de sentimientos (la interacción docente - estudiante se encuentra mediada por aspecto propios del ciclo vital, sociales y culturales) (Ministerio del Trabajo, 2019).

Frente a las demandas de carga mental, es importante referir que la labor docente tiene un alto riesgo por el nivel de atención requerido y por el desarrollo de varias tareas de forma simultánea. Riesgos relacionados también con las demandas cuantitativas, porque la cantidad de trabajo supera el tiempo para el desarrollo de la labor docente: preparación de clases, correcciones de trabajo y evaluaciones; esto sumado al número de estudiantes por grupo, hace que la cantidad de tiempo en ejecución de la tarea sea mayor (Ministerio del Trabajo, 2019).
Al respecto, Restrepo y López (2013), refieren como los docentes universitarios se encuentran expuestos a presiones provenientes de su contexto laboral, como: carga laboral, gestión del talento humano, estilos de dirección, demandas del trabajo y riesgos psicosociales; que afectan la CVL y por ende, la percepción de bienestar frente a su espacio de trabajo, el desempeño laboral, el posicionamiento de la organización y su competitividad.

Para el caso del Departamento de Nariño (Colombia), entre los años 2015 y el primer semestre del 2021, La Federación de Aseguradores Colombianos (FASECOLDA, 2021) da a conocer la estadística de Riesgos laborales, frente al sector educación (riesgo clase 1) y en la cual se evidencia que para educación superior se reportaron 670 accidentes de trabajo y 12 enfermedades calificadas como laborales y 0 muertes. Es importante referir que durante el año 2020 cuando se debió realizar trabajo en casa por parte de los docentes, el reporte de accidentes es de 30, en comparación con el 2019 que se reportaron 86 accidentes laborales. Una posible reflexión frente a las estadísticas se relaciona con la ausencia de protocolos y normatividad específicas para asumir el trabajo en casa, lo cual imposibilitó el proceso, de igual forma, es importante referir que los accidentes laborales se mantienen a lo largo de los años, lo cual lleva a reflexión sobre la importancia y necesidad de seguir trabajando en la cultura de la prevención.

Los reportes brindados por el DANE (2021) en la gran encuesta integrada de hogares en Colombia - GEIH que indaga entre otras variables las dificultades ocurridas como consecuencia de la pandemia generada por COVID - 19, muestra como la situación laboral se ha visto afectada en el caso de los docentes al abordar desde su rol estrategias mediadas por la tecnología, lo cual implicó nuevos aprendizajes y retos en el acto de enseñar. De igual forma, la encuesta permite 
ver como las mujeres reportaron sentirse más estresadas, preocupadas o deprimidas, sintomatologías que se relacionan con la salud mental e incrementan el número de casos de estrés, ansiedad y depresión, y que, a su vez, afectan la salud, el bienestar y la calidad de vida en el trabajo (DANE, 2021).

Todos los aspectos anteriormente revisados refieren la necesidad de abordar aspectos relacionados con la $\mathrm{CVL}$, la cual se empieza a estudiar en la década de los 70 con la intención de humanizar los centros de trabajo, en la década de los 80 la CVL se relaciona con la importancia de gestionar un ambiente de trabajo que aporte en la satisfacción de las necesidades del trabajador debido a que estas tienen impacto en la productividad. En la década de los 90 se propendió por generar ambientes de trabajo que favorezcan la satisfacción laboral, pues estas intervienen en la productividad de las organizaciones, y del 2000 en adelante, se integra aún más la salud, el bienestar del trabajador y el desarrollo integral como elementos importantes de la CVL (Matabanchoy, Betancourth y Calderón, 2015).

De esta manera, la CVL se conforma por dos dimensiones: la objetiva relacionada con condiciones que el trabajo, y la dimensión subjetiva enfocada a la percepción del trabajador (Segurado y Agulló, 2002).

Para este estudio, la CVL se entiende como un concepto que integra muchas variables en el colaborador, respecto a su empleo y su percepción, en cuanto a: soporte institucional, seguridad e integración al puesto de trabajo y satisfacción por el mismo; alcanzando un nivel de bienestar a través de su actividad laboral, desarrollo personal y administración de su tiempo libre (González, Hidalgo, Salazar y Preciado, 2009).

Por lo tanto, la CVL se ha convertido en un pilar para toda organización, que exige un grado de satisfacción y bienestar en la persona, puesto que esta influye en los resultados organizacionales como la consecución de logros y objetivos, el desempeño, la implicación del colaborador con su empleo, entre otros (Blanch, 2011; Huerta et. al, 2012; Pérez y Zurita, 2014; Salas, et.al, 2021). Así, diferentes estudios reportan que, aunque la CVL puede encontrarse en los colaboradores en un nivel adecuado, se presentan otros aspectos importantes como la salud percibida, el nivel de carga laboral, el deterioro de la vida familiar, el estrés y los factores del medio ambiente físico del trabajo, que pueden percibirse de manera desfavorable (Ureña y Castro, 2009; Mariño, Guirado y Matamoros, 2011, Sánchez y Martínez, 2014; Miño, 2016; Salas, et.al, 2021).

Por consiguiente, la CVL se estudia desde las posibles variables que en ella influyen, tales como las características físicas y de ambiente laboral, y aquellos acontecimientos originados en áreas extralaborales que conforman la cotidianidad de los colaboradores, que a su vez, determinan los modos de vida, los comportamientos e interacciones de los individuos, de acuerdo a los parámetros culturales que una sociedad establece en los variados contextos en los que interactúa la persona, como los educativos (Granados, 2011; Figueroa, et. al, 2017; Chaves; et. al, 2017).

En instituciones de educación superior confluyen además un sinnúmero de elementos que se relacionan con la CVL, respeto a los códigos de género que cada sociedad impone y que construyen identidades femeninas y masculinas de las personas (Díaz, 2005), de allí, que se pueda identificar diferencias debido a los Roles de género en todos los ámbitos.

Es así, como la categoría de análisis de género impacta en las relaciones de hombres y mujeres, reforzando en muchos de los casos, los roles asignados tradicionalmente a los sexos (Roles de género), que pueden desencadenar diversas manifestaciones de fuerza, poder, 
discriminación y violencia, como el acosolaboral, la violencia basada en género, y condiciones de inequidad en el trabajo, la asignación de roles, tareas o salarios, entre otras (Connell, 2001, Díaz, 2005; Anaya y Suarez, 2006; González, et. al., 2015; Becerra, 2015; Zambrano, Perugache y Figueroa, 2017, Pérez y Betancourt, 2019).

En este sentido, los roles de género designan funciones, expectativas y normas que se espera que las mujeres y los hombres cumplan en una sociedad, e imponen pautas sobre la forma como deben ser, sentir y actuar los sujetos. Por esta razón, para el presente estudio se entienden los Roles de género como toda verbalización que un grupo social relaciona hacia unas características propias de un individuo de acuerdo a su sexo (Saldívar, et. al. 2015).

Así, las características laborales son distintivas para mujeres y hombres, y se reportan escasos estudios que verifican las diferencias de Roles de género en el contexto laboral y sus implicaciones en la CVL, pese a que elementos propios del género, como los Roles de género, es una de las variables que puede expresar, particularidades en cuanto a la percepción de la CVL y su impacto en el bienestar y la salud de los docentes universitarios (Becerra, 2015; Rodríguez, 2016).

Teniendo en cuenta lo anterior, sumado a las limitadas investigaciones que relacionen la CVL y los Roles de género, se reconoce un vacío en el conocimiento en la temática desarrollada, obstaculizando el diseño de estrategias de premoción y prevención que permitan mejorar la CVL desde una perspectiva de género en docentes universitarios; por lo cual, se propone como objetivo de investigación analizar la relación entre calidad de vida en el trabajo y Roles de género en docentes universitarios.

\section{METODOLOGÍA}

La investigación fue cuantitativa, de tipo descriptivo correlacional con diseño transversal, debido a que su propósito era analizar la relación entre las variables de Calidad de vida en el trabajo con Roles de género, por medio de la aplicación de unos instrumentos en un momento determinado (Hernández, Fernández y Baptista, 2014).

Para este fin, se utilizó un muestreo intencional, contando con la participación voluntaria de 206 docentes (140 (68\%) hombres y 66 (32\%) mujeres), vinculados a una universidad pública de la ciudad de San Juan de Pasto, entre diciembre del 2019 a marzo del 2020.

Para medir la CVL se aplicó el instrumento CVT-GOHISALO de González, Hidalgo, Salazar y Preciado (2009) y para Roles de género la Escala de Roles de Género de Saldívar, et al. (2015).

La CVT-GOHISALO, se compone de 74 ítems distribuidos en siente dimensiones a saber: Soporte institucionaleneltrabajo(SIT), Seguridad en el trabajo (ST), Satisfacción alcanzada a través del trabajo (SAT), Integración al puesto de trabajo (IPT), Bienestar logrado a través del trabajo (BLAT), Desarrollo personal logrado a través del trabajo (DPLT) y Administración del tiempo libre (ATL). El instrumento cuenta con adecuadas evidencias de validez y confiabilidad (Alfa de Cronbach de 0,97) (González, Hidalgo, Salazar y Preciado, 2009).

La Escala de Roles de Género consta de 18 ítems organizados en las siguientes tres dimensiones: Roles femeninos estereotipados, Roles masculinos estereotipados, y Roles tradicionales para mujeres y varones. La escala reporta adecuadas evidencias de validez y confiabilidad (Alfa de Cronbach de 0,89) (Saldívar, et al., 2015). Para la presente investigación, Zambrano, López y Muñoz 
(2021), realizaron la adaptación del instrumento encontrando tres factores a saber:

a) Sistema patriarcal y roles de género femeninos (RolFes): que se ejemplifica en la imposición de unas reglas que se establecen entre los hombres y las mujeres, que muestran a la mujer con mayores capacidades para expresar sus sentimientos y emociones.

b) Sistema patriarcal y roles de género masculinos (RoIMas): que ejemplifica la imposición de unas reglas que se establecen entre los hombres y las mujeres, que muestran al hombre como promiscuo e infiel, fuerte y racional.

c) Sistema patriarcal y roles de género masculinos y femeninos (RolMasyFes): que se ejemplifica en la imposición de unas reglas que se establecen entre los hombres y las mujeres, que se relacionan a la obligación de las mujeres de atender a su pareja y hogar; mientras que para los hombres sobresale su rol protector.

En el instrumento en general se obtuvo adecuadas propiedades psicométricas, con un Alfa de Cronbach de 0,93, y en sus dimensiones: RolFes (Alfa de Cronbach 0,86), RolMas (Alfa de Cronbach 0,89) y RolMasyFes (Alfa de Cronbach 0,829 (Zambrano, López y Muñoz, 2021).

Durante el desarrollo de la investigación, inicialmente, se contó con el permiso de los autores de los instrumentos y se adaptó la Escala de Roles de Género de versión mexicana al contexto colombiano, así como también, tanto la Escala de Roles de Género y el instrumento CVT-GOHISALO se adaptaron de formato (de papel y lápiz a digital); contemplado en todos los casos los lineamientos psicométricos para tal fin. Posteriormente, se generó un proceso de sensibilización e información a los docentes vinculados en la Universidad, sobre la importancia de medir Calidad de vida en el trabajo y los Roles de género, a través de los medios de comunicación institucional, como: periódico, radio, televisión y página web.

Seguidamente, se obtuvo el consentimiento informado de los docentes interesados en apoyar en el estudio, realizando la aplicación de los instrumentos de manera virtual. Finalmente, recogida la información se realizaron los análisis estadísticos respectivos mediante el programa SPSS versión 23. Así, se procedió a describir los niveles de CVL y Roles de género, y a realizar las correlaciones respectivas entre las variables de estudio y sus dimensiones.

Es importante referir, que la investigación contó con el aval del Comité de Ética de la Vicerrectoría de Investigación e Interacción Social de la Universidad en la cual se desarrolló el estudio, contemplado los lineamientos establecidos en el Código Deontológico y Bioético de la Ley 1090 del 2006 (por la cual se reglamenta el ejercicio de la psicología en Colombia), y la Resolución 8430 de 1993 (en la cual se establecen las normas científicas técnicas y administrativas para la investigación en salud).

\section{RESULTADOS}

A continuación, se presentan los resultados descriptivos de Calidad de vida en el trabajo y Roles de género, seguido de la relación entre dichas variables y sus dimensiones.

\section{CALIDAD DE VIDA EN EL TRABAJO}

Para la mayoría de los docentes universitarios la CVL se encuentra en un nivel medio $(41,26 \%)$, seguido de alto $(33,98 \%)$ y bajo $(24,76 \%)$. En cuanto a las dimensiones del instrumento, se encuentra en su mayoría la SIT $(38,8 \%)$, ST $(35,9 \%)$, SAT $(35,9 \%)$, DPL $(36,4 \%)$ y ATL $(45,1 \%)$ en percepción baja; la IPT en percepción media $(36,9 \%)$, y la BLAT en percepción Alta $(43,2 \%)$. 


\section{Roles de género}

Para la mayoría de los docentes universitarios se identifica respecto a Roles de género una percepción considerablemente estereotipada $(26,7 \%)$, seguido de una percepción estereotipada baja $(25,2 \%)$ y una percepción altamente estereotipada de los roles de género $(25,2 \%) y$, por último, una percepción moderadamente estereotipada $(22,8 \%)$.

Asimismo, en las dimensiones del instrumento, se observa que la mayoría de docentes presenta en RolFes una percepción estereotipada baja en roles femeninos (30\%), en RolMas una percepción estereotipada baja en roles masculinos $(29,1 \%)$, y en RolMasyFes una percepción considerablemente estereotipada de roles tradicionales para mujeres y varones $(32,5 \%)$.

\section{Relación entre Calidad de vida en el trabajo y Roles de género}

Para analizar la relación entre las puntuaciones obtenidas a partir de los instrumentos aplicados de CVL y Roles de género, previamente se comprobó mediante la prueba de KolmogorovSmirnov que la variable de CVL tiene una significancia de 0,0074 , y la variable de Roles de género una significancia de 0.001 ; por lo que dichas variables presentan una distribución no normal. De esta forma, se correlacionan las dos variables objeto de estudio con Rho de Spearman, obteniendo un coeficiente de -0.188 $(p<.05)$, lo que muestra una relación negativa y baja entre CVL con Roles de género.

Por su parte, respecto a las correlaciones entre las dimensiones de CVL y Roles de género, se encuentra una relación negativa y baja entre las mismas (ver tabla 1).

Tabla 1. Correlaciones entre las dimensiones de Calidad de vida en el trabajo y Roles de género.

\begin{tabular}{|l||c|c|c|c|c|c|}
\hline \multicolumn{1}{|c|}{ Variable } & \multicolumn{1}{c|}{ RoIFes } & \multicolumn{2}{c|}{ RoIMas } & \multicolumn{3}{c|}{ RoIMasyFES } \\
\hline SIT & -0.080 & & -0.117 & & -0.012 & \\
\hline ST & -0.163 & ${ }^{*}$ & -0.138 & ${ }^{*}$ & -0.099 & \\
\hline SAT & -0.217 & ${ }^{* *}$ & -0.206 & ${ }^{* *}$ & -0.170 & \\
\hline IPT & -0.164 & ${ }^{*}$ & -0.203 & ${ }^{* *}$ & -0.138 & \\
\hline BLAT & -0.216 & ${ }^{* *}$ & -0.271 & ${ }^{* * *}$ & -0.208 & \\
\hline DPLT & -0.169 & ${ }^{*}$ & -0.195 & $* *$ & -0.141 & \\
\hline ATL & -0.098 & & -0.071 & & -0.011 & \\
\hline
\end{tabular}

Nota: Calidad de vida en el trabajo: Soporte institucional en el trabajo (SIT), Seguridad en el trabajo (ST), Satisfacción alcanzada a través del trabajo (SAT), Integración al puesto de trabajo (IPT), Bienestar logrado a través del trabajo (BLAT), Desarrollo personal logrado a través del trabajo (DPLT) y Administración del tiempo libre (ATL).

Roles de género: Roles femeninos estereotipados (RolFes), Roles masculinos estereotipados (RolMas) y Roles tradicionales para mujeres y varones (RolMasyFES). 


\section{DISCUSIÓN}

La presente investigación tuvo como objetivo analizar la relación entre Calidad de vida en el trabajo y Roles de género en docentes universitarios, es así, que a continuación se presenta la discusión sobre lo reportado en CVL, Roles de género y finalmente su correlación.

La CVL busca incrementar la productividad como mejorar el bienestar de los colaboradores y su entorno (Salas, et. al, 2021). En el contexto universitario, los docentes enfrentan diversas condiciones laborales cambiantes en coherencia a las expectativas y su contraste con la realidad, políticas educativas y gestión organizacional. Lo anterior, afecta las actividades que lideran los educadores, así como también, su percepción sobre las condiciones de trabajo a las cuales están expuestos (Hernández, Morales y Arriaga, 2017).

Así, en el presente estudio se reporta que la mayoría de los docentes universitarios su CVL se encuentra en un nivel medio y alto lo que implica que en promedio es adecuada. Esto evidencia que desde la percepción del docente existe satisfacción en relación al apoyo institucional que recibe, la seguridad e integración al puesto de trabajo y su desarrollo personal (González, Hidalgo, Salazar y Preciado, 2009). Estudios como los de Huertas, et.al. (2012) reportan como la CVL percibida en un buen nivel contribuye al resultado de las organizaciones, sin embargo, en la investigación se encontró que $24,76 \%$ de los docentes perciben una CVL baja, lo que implica la posibilidad de seguir fortaleciendo aspectos relacionados con la gestión de los riegos laborales y la posibilidad de crear ambientes de trabajo satisfactorios que favorezcan la salud y el bienestar (González, Hidalgo, Salazar y Preciado, 2009).

Estos datos permiten reflexionar sobre la importancia y necesidad de continuar trabajando la CVL desde los programas de Seguridad y
Salud en el Trabajo, considerando, además, como el estudio de Salas, et. al. (2021), indica que la población docente es uno de los sectores en los que más se ha trabajado la CVL, dejando la evidencia de la necesidad de considerarla, dado el impacto de la misma en el desarrollo de las funciones de los docentes y la satisfacción por el trabajo (Pérez y Zurita, 2014).

Asimismo, respecto a las dimensiones de CVL, se encuentra en los docentes que la SIT, ST, SAT, DPL y ATL presentan una percepción baja. Esto refiere que el docente evidencia dificultades en la realización de las tareas, aspecto que impacta en su motivación y puede llegar a percibir que el trabajo le ha disminuido la capacidad física y emocional. Lo anterior, lo ratifica Alves (2013) al mostrar una relación entre CVL y motivación, y como el nivel académico con el que se enseña, la motivación por el rol docente y la satisfacción con condiciones laborales son predictores de una mejor CVL.

Por otra parte aspectos relacionados con modalidades de contratación hace evidente que el docente asuma otros compromisos laborales, lo cual hace que la dedicación sea parcial impactando en la cobertura de necesidades personales y en la percepción de igualdad por parte de los docentes, esto mismo lo señala Garrido, Uribe y Blanch (2011) que resaltan la afectación de docentes universitarios por las condiciones laborales, en el que variables como la contratación temporal, entre otras, han influido en la salud y bienestar del docente.

La afectación de la salud por la exposición a riesgos laborales derivados de las condiciones de trabajo y la inadecuada distribución del tiempo, hace que el trabajo deba llevarse a casa afectando otras dimensiones de la vida personal y familiar, como también su satisfacción laboral (González, Hidalgo, Salazar y Preciado, 2009). Estudios como los de Blanch (2012) permite ver que la sobre carga de trabajo, genera una experiencia de saturación acompañada 
de percepción de falta de tiempo, lo que se configura también como factores de riego psicosocial. El trabajo de Miño (2016) permite visualizar que situaciones relacionadas con la cantidad de trabajo, hacen difícil terminar la jornada laboral y, por tanto, la dificultad para desconectarse del trabajo, impactando en la esferas extra laborales relacionadas con la familia y actividades diferentes a la laboral.

Respecto a la dimensión IPT se encuentra en percepción media, representando que el docente integra equipos de trabajo y muestra su capacidad para crear ambientes de trabajo satisfactorios y la BLAT en percepción Alta, lo que significa que el docente se siente satisfecho por los aportes que desde su profesión hace a la sociedad (González, Hidalgo, Salazary Preciado, 2009). Lo mismo se refiere en el estudio de Blanch (2014), resaltando como factor protector la valoración de las condiciones sociales del trabajo y que, si bien la gestión del trabajo se encuentra saturada por factores de riesgo psicosociales, las estrategias de afrontamiento personal tienen efectos organizacionales.

Por otra parte, es importante referir como los Roles de género están presentes en los diferentes contextos en los cuales está inmersa la persona, que en el contexto universitario se puede observar la relación que se establece entre hombres y mujeres desde su rol docente. Dichas relaciones pueden estar mediadas por construcciones que tradicionalmente han sido aceptadas por una sociedad patriarcal, que según Zambrano, Perugache y Figueroa (2017), los Roles de género no se construyen de manera automática, sino que se elaboran en relación con el otro, naturalizándose en las prácticas cotidianas, al basarse en responsabilidades y tareas que se cumplen dentro de la institución universitaria y las actitudes que cada persona asume con relación a su rol de hombre, mujer, profesor, profesora, padre, madre, entre otros.
En este estudio se observa en los docentes una percepción considerablemente estereotipada y altamente estereotipada de los roles de género femeninos, en contraste con una percepción estereotipada baja y una percepción moderadamente estereotipada. Estos resultados muestran que más de la mitad de los docentes universitarios que contestaron el instrumento tienen una percepción considerable y altamente estereotipada en Roles de género, aspectos que llaman la atención, puesto que se podría suponer que aún se presentan ciertas reglas entre los hombres y las mujeres que permiten mostrar actitudes diferenciadas según el sexo, en aspectos como: la sexualidad, expresión de sentimientos, quehaceres del hogar, entre otros (Saldívar, et al., 2015).

Específicamente, en las dimensiones del instrumento de roles de género, se identifica que la mayoría de docentes presenta en RolFes una percepción estereotipada baja en roles femeninos, lo que muestra que para los docentes no necesariamente la mujer, presenta mayores capacidades para expresar sus sentimientos y emociones en comparación a los hombres. Igualmente, en la dimensión RolMas, hay una percepción estereotipada baja en roles masculinos, en el que los docentes no necesariamente perciben a la mujer con mayores capacidades para expresar sus sentimientos, emociones y cariño.

No obstante, en la dimensión RolMasyFes, para los docentes existe una percepción considerablemente estereotipada de roles tradicionales para mujeres y varones, representadas en la posibilidad de percibir una obligación de las mujeres de atender a sus pareja, realizar los quehaceres domésticos y sus necesidad de convertirse en madre; en comparación a los hombres, en los que su exige su rol protector y de habilidades para el cortejo amoroso (Saldívar, et al., 2015; Zambrano, López y Muñoz, 2021). 
Lo anterior, no influye únicamente en la relación que se establece entre docentes hombres y mujeres, sino entre los docentes con otros miembros de la comunidad educativa, como los estudiantes. Así, estudios como Páramo (2010) y Espinoza y Taut (2016) encontraron de manera diferenciada como los docentes generaron expectativas y desarrollaron su práctica pedagógica con sus estudiantes, de acuerdo al sexo de los mismos, interactuando, por ejemplo, en menor medida con las estudiantes que con los estudiantes.

Este contexto muestra, que los Roles de género permiten que las personas construyan expectativas $\mathrm{y}$, por lo tanto, los individuos se comporten en coherencia a lo que se espera de un hombre y una mujer, independientemente del rol docente o de estudiante; puesto que la categoría de género es transversal en toda profesión, actividad u oficio. En palabras de Lamas (1994), una sociedad establece y significa el mundo en función del sexo/género, al construir normas y mandatos según la cultura, clase social, grupo étnico, entre otras; determinando y reglamentando comportamientos femeninos o masculinos.

Finalmente, al establecer la relación entre CVL y Roles de género, así como también entre las dimensiones de los dos instrumentos, se encuentra una baja relación entre todas las variables, lo cual parece indicar que la CVL y Roles de género (incluidas sus dimensiones) no varían entre ellas, y por lo tanto, su comportamiento depende en función de cada una por separado.

Este resultado encontrado llama la atención, considerando que la teoría muestra como los Roles de género en el medio laboral tanto en su contexto intra y extralaboral, impactan en la salud, bienestar y calidad de vida de los colaboradores (González, Hidalgo, Salazar y Preciado, 2009; González, 2011; Figueroa, et. al, 2017; Chaves; et. al, 2017; Salas, et. al, 2021).
Asimismo, Pérez (2012) refiere que pesar de los cambios históricos y socioeconómicos, aún se registra una división tradicional del trabajo entre hombres y mujeres.

Del mismo modo, en la búsqueda realizada no se reportan estudios que relacionen CVL y Roles de género en docentes universitarios, más aún, la investigación Relación entre género y calidad de vida laboral en profesionales de salud de González, et.al. (2014), encuentra la relación entre las variables, reportando una desigualdad de oportunidades entre hombres y mujeres, a pesar de que las mujeres se muestras más satisfechas en el desarrollo personal alcanzado en trabajo y en la administración de su tiempo libre.

Buscando explicar los resultados obtenidos en esta investigación, se puede inferir posibles causas respecto a cómo afectan las variables de CVL y Roles de género en docentes universitarios.

Inicialmente, es importante referir como los Roles de género establecen códigos y pautas sociales y culturales presentes en los diferentes contextos de interacción de las personas (Saldívar, et. al., 2015); mientras que la CVL, muestra el grado de satisfacción y bienestar más específico en el entorno laboral donde se encuentra la persona (González, Hidalgo, Salazar y Preciado, 2009).

Por lo tanto, en especial el instrumento de Roles de género implementado en el estudio, mide este atributo orientado a estereotipos tradicionalmente trasmitidos en la sociedad, como: delegar a la mujer la capacidad de cuidar a los enfermos, el quehacer doméstico, la atención del esposo, la realización como madres, la facilidad de expresión de sentimientos, entre otros; y al hombre, se lo asocia con dificultades para ser cariñoso, la posibilidad de tener más parejas sexuales, de cortejar y ser infiel (Saldívar, et. al., 2015). Dichos ítems, puede tener menor sensibilidad en los docentes 
universitarios, puesto que tanto hombres como mujeres, muestran un importante desarrollo académico, investigativo y profesional; además, de las capacitaciones, cursos y eventos culturales a los cuales hacen parte, que exigen a los docentes actualización y movilización de actitudes frente a la reivindicación de derechos, inclusión y respeto por la diversidad.

Así, las docentes del siglo XXI en comparación a sus compañeros, pese a que no han conseguido similar acceso a cargos académicos y directivos en el ámbito universitario; legal y socialmente en el contexto universitario, se reconoce la igualdad entre hombres y mujeres en su rol docente (Hernández, García y Sánchez, 2004). Sin embargo, se puede suponer que, en este estudio, los Roles de género construidos por los docentes universitarios no únicamente se basan en estereotipos tradicionales, sino que estos de manera automática se adaptan y otorgan según la distribución de responsabilidades y tareas dentro de la institución (Zambrano, Perugache y Figueroa, 2017).

Por consiguiente, González y Mora (2014), indican como en las universidades se relacionan los factores culturales, sociales, psicológicos, filosóficos, ideológicos, históricos, familiares, económicos y políticos; que afectan positiva o negativamente la interacción entre sus actores respecto a la transformación de sus comportamientos y actitudes. Por lo tanto, quizás en los docentes universitarios con los que se trabajó en esta investigación, no intervienen únicamente los Roles de género tradicionales, sino que se pueden presentar otros en relación a la desigualdad de oportunidades al competir hombres y mujeres académica e investigativamente, puesto que las responsabilidades familiares $y$ tareas domésticas, supone un mayor esfuerzo para las profesoras al tener que mediar entre trabajo, familia y tiempo libre (Hernández, García y Sánchez, 2004).
Por su parte, de acuerdo al contexto anteriormente mostrado, es posible que la CVL se vea también afectada de manera particular, puesto que independientemente de los Roles de género estereotipados presentes en el contexto universitario, las docentes cuenten con un trabajo que les permite generar una percepción de bienestar y satisfacción. Esto hace que se naturalice o tolere prácticas discriminatorias 0 de violencia en relación al género (Zambrano, Perugache y Figueroa, 2017); que según Cutuli (2017), conduce a una precariedad del trabajo, al desconocer los aspectos inmateriales del mismo y el esfuerzo físico que involucra su labor al mediar entre ser madre, esposa, estudiante, trabajadora, entre otras.

\section{CONCLUSIONES}

La CVL y Roles de género son dos categorías de estudio muy importantes y aunque en el presente estudio no se evidenció relación, es muy necesario considerar su inclusión, mediante la creación de estrategias que desde recursos humanos y seguridad y salud en el trabajo se construyan, con el fin de aportar en la humanización de los centros de trabajo, en la salud y bienestar de los docentes, como un aspecto relevante que impacta en el adecuado desempeño de la función docente.

Aunque el rediseño de condiciones de trabajo obedece a un tema estructural que se escapa del manejo que los niveles directivos de las instituciones de educación superior desean y esperan para sus docentes, se hace necesario mostrar la necesidad de continuar trabajando por la calidad de vida de los docentes y su impacto en el acto de enseñar desde su rol de género.

Es importante tener presente los factores de riesgo psicosocial a los que se exponen los docentes, ya que esto aporta en la compresión de la CVL y los posibles programas de intervención que se desarrollen en cumplimiento de uno de los objetivos de desarrollo sostenible, 
relacionado con salud y bienestar para los trabajadores.

Se recomienda para futuros estudios relacionar la CVL y los Roles de género con otros instrumentos que puedan ser más sensibles a las condiciones culturales, educativas, económicas y sociales de los docentes universitarios. Así como también, complementar el estudio desde una mirada cualitativa, que permita acercarse a las actitudes y percepciones frente a las variables objeto de estudio de cada actor involucrado en el contexto universitario (docentes, estudiantes, administrativos y directivos).

\section{REFERENCIAS BIBLIOGRÁFICAS}

Alves, C. (2013). Motivación y calidad de vida y satisfacción del profesional docente. España: Universidad de Cádiz

Anaya, D. y Suarez, J. (2006). La satisfacción laboral de los profesores en función de la etapa educativa, del género y de la antigüedad profesional. Revista de Investigación Educativa, 24(2), 541-556.

Becerra, C. (2015). Calidad De Empleo Con Enfoque De Género: Propuesta De Un Marco Conceptual. Cuestiones de género: de la igualdad y la diferencia, 10, 194-224. doi.org/10.18002/ cg.v0i10.1177

Blanch, J. (2011). Condiciones de Trabajo y Riesgos Psicosociales Bajo la nueva Gestión. FOCAD, Universidad Autónoma de Barcelona.

Blanch, J. (2012). Trabajar y bienestar. España: Universidad Abierta de Cataluña.

Blanch, J. (2014). Calidad de vida laboral en hospitales y universidades mercantilizados. Papeles del Psicólogo, 35(1), 3-13.
Chaves, L., Grijalba, M., Bárcenas, D., Matabanchoy, S., \& Zambrano, C. (2017). Actitudes hacia la calidad de vida laboral en trabajadores control de vía de transporte urbano. Tendencias, 18(2), 69-85. doi.org/10.22267/ rtend. 171802.77

Clavijo, D. y Balaguera, A. (2020). La calidad y la docencia universitaria: algunos criterios paras su valoración. Revista Investigación. Desarrollo en Innovación, 11(1), 127 - 139. doi.org/. $19053 / 20278306$.v11. $\mathrm{n} 1.2020 .11688$

Connell, R. (2001). Educando a los muchachos: nuevas investigaciones sobre masculinidad y estrategias de género para las escuelas. Nómadas (Col), 14, 156-171.

Cutuli, R. (2017). Género y trabajo emocional. Los fundamentos de la precariedad en el nivel inicial. Mar del Plata, Provincia de Buenos Aires. Trabajo y Sociedad, 28, 37-54.

DANE (2021). Nota estadística salud mental en Colombia: un análisis de los efectos de la pandemia. Recuperado de https:// www.dane.gov.co/index.php/serviciosal-ciudadano/servicios-informacion/ serie-notas-estadisticas

Díaz, M. (2005). La perspectiva de género en la formación del profesorado de música REICE. Revista Iberoamericana sobre Calidad, Eficacia y Cambio en Educación, 3(1), 570-577.

Espinoza, A. y Taut, S. (2016). El Rol del Género en las Interacciones Pedagógicas de Aulas de Matemática Chilenas. Psykhe (Santiago), 25(2), 1-18. doi.org/10.7764/ psykhe.25.2.858 
FASECOLDA. (2021). Riesgos Laborales. Estadísticos del Ramo. Recuperado de https://sistemas.fasecolda.com/rldatos/ Reportes/xClaseGrupoActividad.aspx

Figueroa, HA, Bastidas, C., Gonzales, W, Zambrano, C., Matabanchoy, S. (2017). Propiedades psicométricas de la Escala de Evaluación de Calidad de Vida Laboral en el sector transporte urbano. Univ. Salud, 19(3), 330-339. doi. org/10.22267/rus.171903.95

Garrido, J., Uribe, A., Rodríguez, A.M., \& Blanch, J. (2011). Riesgos psicosociales desde la perspectiva de la calidad de vida laboral. Acta Colombiana de Psicología,2(14), 27-34.

González, F. y Mora, B. (2014). Características de la violencia de género en la Universidad de Valencia. Escritos de Psicología 7(2), 36-43.

González, M. (2011). Salud laboral género: Apuntes para la incorporación de la perspectiva de género en el ámbito de la prevención de riesgos laborales. Medicina y Seguridad del Trabajo, 57(1), 89-114. doi.org/10.4321/S0465$546 \times 2011000500007$

González, R., Santacruz, G., León, S., Contreras, M., Aldrete, M., Hidalgo, B. y Barrera, J. (2015). Relación entre género y calidad de vida laboral en profesionales de salud. Psicogente,18(33), 52-6. doi. org/10.17081/psico.18.33.55

Granados, I. (2011). Calidad de Vida Laboral: Historia, Dimensiones y Beneficios. IIPSI, 14(2). 271 - 276. doi.org/10.15381/ rinvp.v14i2.2109
Hernández, A., García, A. y Sánchez, M. (2004). El profesorado universitario desde una perspectiva de género. Revista Educación, 28(2),105-143.

Hernández, R., Fernández, C. y Baptista, M. (2014). Metodología de la investigación. México: Mc Graw Hill.

Huerta, P., Pedraja, L., Contreras, S. y Almodóvar, P. (2012). Calidad de Vida Laboral y su influencia sobre los resultados empresariales. Revista de Ciencias Sociales, 17(4). 658-676. doi. org/10.31876/rcs.v17i4.25593

Lamas, M. (1994). Cuerpo: diferencia sexual y género. Debate Feminista, 10, 1 - 31. doi.org/10.22201/ cieg.2594066xe.1994.10.1792

Mariño, A., Guirado, A. y Matamoros, Y. (2011). La calidad de vida laboral en la Facultad de Ciencias Económicas de la Universidad de las Tunas. Cuadernos de educación y desarrollo, 3(26). 5-28.

Matabanchoy, S., Calderón, G., y Betancourt, S. (2014). Aproximaciones a la reflexión sobre la calidad de vida laboral. En Orejuela, J. (Ed). Psicología de las organizaciones y del trabajo (Pág. 447-465). Cali, Colombia, Editorial Bonaventurana.

Ministerio del Trabajo. (2007). Primera Encuesta Nacional de Condiciones de Salud y trabajo en el Sistema General de Riesgos profesionales. Recuperado de https://www.minsalud.gov.co/ riesgosProfesionales/Documents/ ENCUESTA\%20SALUD_RP.pdf

Ministerio del Trabajo. (2013). Informe Ejecutivo de la Segunda Encuesta Nacional de Condiciones de Seguridad y Salud en el Trabajo en el Sistema General 
de Riesgos. Recuperado de https:// fasecolda.com/cms/wp-content/ uploads/2019/08/ii-encuesta-nacionalseguridad-salud-trabajo-2013.pdf

Ministerio del Trabajo. (2019). Prácticas de trabajo saludable para educadores: Protocolo de intervención de factores psicosociales en el sector educativo. Recuperado de https:// sttp.laequidadseguros.coop/2020/arlnoviembre/07.\%20PR\%20sector\%20 educativo.pdf

Miño, A. (2016). Calidad de vida laboral en docentes chilenos. Summa Psicológica UST, 13(2). 45-55. doi. org/10.18774/448x.2016.13.256

Páramo, P. (2010). Las representaciones de genero en profesores universitarios. Estudios pedagógicos (Valdivia), 36(2), 177-193. doi.org/10.4067/S071807052010000200010

Pérez, A. y Betancourt, M. (2019). El enfoque de género desde la formación docente y su relación con la ciencia, la tecnología y la sociedad. Revista Boletín Redipe, 8(3), 200-208. https://doi.org/10.36260/rbr. v8i3.713

Pérez, D. y Zurita, R. (2014). Calidad de Vida Laboral en trabajadores de Salud Pública en Chile. Salud \& Sociedad, 5(2). 172-180. doi.org/10.22199/ s07187475.2014.0002.00002

Pérez-Baleón, G. (2012). Desigualdades de género en el inicio de la vida laboral estable. Papeles de población, 18(72), 213-246.
Restrepo, F. y López, A. (2013). Percepciones del entorno laboral de los profesores universitarios en un contexto de reorganización flexible del trabajo. Cuadernos de Administración, 29(49), 55-63.

Rodríguez, B. (2016). Condiciones de trabajo, satisfacción laboral y calidad de vida laboral en educación y sanidad (tesis doctoral). Universidad Miguel Hernández de Elche, España.

Salas, M., Basante, Y., Zambrano, C., Matabanchoy, S. y Narváez, A. (2021). Concepciones sobre Calidad de Vida Laboral en las organizaciones. Informes Psicológicos, 21(2), pp. 209-227. doi. org/10.18566/infpsic.v21n2a13

Saldívar, A., Díaz, R., Reyes, N., Armenta, C., López, F., Moreno, M., Romero, A., Hernández, J. y Domínguez, M. (2015). Roles de Género y Diversidad: Validación de una Escala en Varios Contextos Culturales. Acta de Investigación Psicológica, 5(3), 2124-2147. doi:10.1016/s2007-4719(16)30005-9

Sánchez, C. y Martínez, S. (2014). Condiciones de trabajo de docentes universitarios, satisfacción, exigencias laborales y daños a la salud. Salud de los Trabajadores, 22(1), 19-28.

Segurado, A., y Agulló, E. (2002). Calidad de vida laboral: hacia un enfoque integrador desde la psicología social. Psicothema, 14(4), 828-836.

UNESCO. (1998). Declaración mundial sobre la educación superior en el siglo XXI. Educación y sociedad, 9(2), 97-113.

Ureña, P. y Castro, C. (2009). Calidad de vida, sentido de coherencia y satisfacción laboral en profesores(as) de colegios 
técnicos en la Dirección Regional de Heredia. Revista Electrónica Educare, 13(1). 71-87. doi.org/10.15359/ree.131.6

Zambrano, C. López, D. y Muñoz, D. (en prensa). Propiedades psicométricas de la adaptación escala de roles de género en docentes universitarios. Revista de educación superior.

Zambrano, C., Perugache, A. y Figueroa, J. (2017). Manifestaciones de la violencia basada en género en docentes universitarios. Psicogente, 20(37), 146159. doi.org/10.17081/psico.20.37.2424 